\title{
The prevalence of non-communicable diseases among academic staff members in rural university, $\mathrm{SA}$
}

\author{
Lindelani Fhumudzani Mushaphi and Sedzani Madala \\ University of Venda, Thohoyandou, South Africa
}

\section{Abstract}

Background: Globally non-communicable diseases (NCDs) remain unacceptably high amongst adult population and its prevalence increases yearly.

Aim: The aim of the study was to determine the prevalence of non-communicable diseases

Methods: The study design used was cross-sectional. The study population was academic staff members, whereas the targeted population was UNIVEN academic staff members. Convenience sampling was used to select participants. Data was collected using a questionnaire. Anthropometric, clinical and biochemical measurements were also taken. Descriptive data statistics were used to interpret data. Percentage and tables were used to present data.

Results: Almost all participants (93\%) were Africans while very few were from other race such as European and Asian. More than one third $(39.2 \%)$ of the participants had no family history of diseases, $23.8 \%$ had family history of hypertension and diabetes. The majority of the participants $(82.3 \%$ ) did not suffer from any chronic diseases of lifestyle. About $7.7 \%$ had SBP between $140-159 \mathrm{mmHg}$, while very few participants $(5.4 \%)$ had $>/=160 \mathrm{mmHg}$. About $13.8 \%$ had DBP between $90-99 \mathrm{mmHg}$, while very few participants $(5.4 \%)$ had $>/=100 \mathrm{mmHg}$. About $43.8 \%$ of the participants were pre-diabetic. Nearly two thirds $(66.2 \%)$ of the participants fell within high borderline of cholesterol, while $13.8 \%$ of participants had normal total cholesterol. About $40 \%$ of the participants were overweight, $20.7 \%$ were obese class I, about $43.8 \%$ of the participants had low waist circumference, more than one thirds (35.4\%) had high waist circumference.

Conclusion: The study revealed that majority of academic staff members are at risk of developing NCDs. Nearly half of participants $(49.3 \%)$ their systolic blood pressure was above normal and the diastolic blood pressure was $48.4 \%$. The blood glucose levels showed that $43.8 \%$ of participants were pre-diabetic and $13.1 \%$ were diabetic. Furthermore, majority of participants $(83.1 \%)$ showed that the cholesterol level was above borderline and had high cholesterol. Majority of participants $(80.8 \%)$ were overweight and obese.

\section{Conflict of Interest}

No conflict of interest 